\title{
Dynamical Analysis of Rumor Spreading Model considering Node Activity in Complex Networks
}

\author{
Liang'an Huo $\mathbb{D}$, Fan Ding, Chen Liu, and Yingying Cheng \\ Business School, University of Shanghai for Science and Technology, Shanghai 200093, China \\ Correspondence should be addressed to Liang'an Huo; huohuolin@yeah.net
}

Received 18 November 2017; Revised 13 August 2018; Accepted 30 September 2018; Published 12 November 2018

Academic Editor: Michele Scarpiniti

Copyright (C) 2018 Liang'an Huo et al. This is an open access article distributed under the Creative Commons Attribution License, which permits unrestricted use, distribution, and reproduction in any medium, provided the original work is properly cited.

\begin{abstract}
The dynamic models are proposed to investigate the influence node activity has on rumor spreading process in both homogeneous and heterogeneous networks. Different from previous studies, we believe that the activity of nodes in complex networks affects the process of rumor spreading. An active node can have contact with all the nodes it directly links to, while an inactive node could only interact with its active neighbors. We explore the joint effort of activity rate, spreading rate and network topology on rumor spreading process by mean-field equations and numerical simulations, which reveals that there exists a critical curve consisting of critical activity rate and spreading rate; meanwhile, activity rate and spreading rate both have influence on the final rumor spreading scale.
\end{abstract}

\section{Introduction}

Rumors play an important role in human affairs. Especially, at first it shakes human's opinions to affect their behaviors in financial market [1] and then causes economic loss and unnecessary public panic $[2,3]$. Owing to such results, spreading dynamics attracts scholars' eyes from many fields after the pioneer works [4-6], which may help us to better understand rumor spreading process along with laying down effective strategies to minimize rumor spreading size.

Due to the similar spreading process between disease spreading and rumor spreading, epidemic models have been widely applied to reveal the rumor spreading process [7]. Although disease spreading and rumor spreading possess some similar properties, it is still difficult to analyze them under a unified framework or use analogous model. Daley and Kendall introduced a classical rumor spreading model (DK model) in 1964, where the population was divided into three groups, ignorant, spreader, and stifler, assuming that the transition between two groups satisfies a certain mathematical probability distribution $[8,9]$. Then DK model was developed and a mathematical model was applied by Maki and Thomson [10] and Murray to study the rumors [11]. Although the above studies are mainly focused on the theoretical analysis, complex network theory provides the methods for settling several problems, such as the differences of spreading rate among different individuals as well as differences of spreading law in different topology structures of social networks, which all push the study on rumor spreading forward [12-14]. Zanette has firstly proposed a rumor spreading model on smallworld network by applying complex network theory after studying the rumor spreading process $[15,16]$. Besides, some scholars found that heterogeneity of network exerted a priory influence on mechanism of rumor spreading when studying the randomness applied to the DK model on scale-free (SF) network, showing that the heterogeneity of the network exacts primary influence on mechanism of rumor spreading process $[2,17,18]$. What is more, it is also found that spreading dynamics will be affected by networks structure; specifically, network with heterogeneity can remove threshold of disease outbreak $[15,19,20]$. Boccalettia et al. review the major concepts and results recently achieved in the study of the structure and dynamics of complex networks [21]. Later, Zhou.et al. took into account the influence of topological structure and distinguished the unequal footings between the father and the other neighboring nodes [22]. Deng et al. took forgetting and remembering rates into account when studying rumor spreading model [23]. Huo et al. took the prevalence of media into consideration when studying rumor spreading model [24]. When Xia et al. studied the rumor propagation 
model, they took hesitating mechanism into consideration on both small-world network and scale-free network [25]. Li et al. took government punishment into account when studying rumor spreading model [26] and Hu et al. established a rumor spreading model considering the proportion of wiseman in the crowd.

Similar to the fact that some scholars believe that the scientific knowledge, hesitating mechanism along with forgetting and remember rates has important effects on final rumor spreading size. We also think that individual activity plays an important role in rumor spreading process. Specifically, the chances to get the rumor and the probability to spread the rumor in these models have strong relations with the frequency of individuals logging in the network, which is what we here called individual activity. It assumes that the activities of adjacent nodes were heterogeneous and they can be expressed by an exponential distribution, which may lead to Poissonian activity patterns. Besides, the above models all conform to the following three assumptions: firstly, all node activities has the same interval, secondly, the response time of these nodes is greatly heterogeneous and, finally, all kinds of human activities would lower the time of information transmission [27-29]. However, it has been found that node activity has a significant effect on contagion processes [30]. Liu et al. took node activity into consideration when studying disease transmission in heterogeneous networks [31]. Huo et al. incorporated the activity of spreaders in homogeneous network while studying rumor transmission model [27].

Based on Liu's and Huo's works, in this paper, we also believe that individual activity exerts influence on rumor spreading process. A simple model is established to study the impacts activity rate and network topology on rumor spreading process. An activity rate is introduced to measure the contact between nodes. Similar to Liu's assumption [31], in every interval, an active node will interact with the nodes it links to directly, while an inactive node could only interact with active nodes it links to directly. The difference is that Liu et al. discussed about the effect of activity on disease spreading, while this paper analyzes about the effect of activity on rumor spreading, taking the stifling rate into consideration. In this model, the active spreader can interact with spreaders and stiflers in active or inactive state and the inactive rate can only interact with active spreaders and stiflers. It is found that the threshold of rumor spreading will be reshaped by node activity after using mean-field method to analyze the rumor dynamics and validate our analysis by numerical simulation on both homogeneous and heterogeneous networks; on top of that, it is also concluded that the topology of network and node activity exert influence on the final rumor size and transmission speed.

We structure this paper as follows: in Section 2, ISR rumor spreading model with node activity is described, then the formulation of the mean-field analytic results of this model will be presented on heterogeneous networks in Section 3, and in Section 4, we give steady analysis of our model on both homogeneous networks and heterogeneous networks, which will be validated by the numerical simulation in Section 5. Finally, we will sum up our analysis in Section 6.

\section{ISR Rumor Spreading Model}

In this model, superscript $a$ denotes active state and $d$ inactive state. The nodes can be in one of the following six states: active ignorant $\left(I^{a}\right)$, inactive ignorant $\left(I^{d}\right)$, active spreader $\left(S^{a}\right)$, inactive spreader $\left(S^{d}\right)$, active stifler $\left(R^{a}\right)$, and inactive stifler $\left(R^{d}\right)$. We give an activity rate $\alpha$ to each node. That is to say, a node could be in active state with rate $\alpha$ and in inactive state with rate $1-\alpha$. The reaction process can be schematically represented by the following:

(i) Behavior state change

$$
\begin{aligned}
& I^{a} \stackrel{1-\alpha}{\longrightarrow} I^{d}, \\
& S^{a} \stackrel{1-\alpha}{\longrightarrow} S^{d}, \\
& R^{a} \stackrel{1-\alpha}{\longrightarrow} R^{d} \\
& I^{d} \stackrel{\alpha}{\rightarrow} I^{a}, \\
& S^{d} \stackrel{\alpha}{\rightarrow} S^{a}, \\
& R^{d} \stackrel{\alpha}{\rightarrow} R^{a}
\end{aligned}
$$

(ii) Active spreading

$$
\begin{aligned}
& I^{a}+S^{a} \stackrel{\lambda}{\rightarrow} S^{a}+S^{a}, \\
& I^{a}+S^{d} \stackrel{\lambda}{\rightarrow} S^{a}+S^{d} \\
& S^{a}+S^{a} \stackrel{\beta}{\rightarrow} R^{a}+S^{a}, \\
& S^{a}+S^{d} \stackrel{\beta}{\rightarrow} R^{a}+S^{d} \\
& S^{a}+R^{a} \stackrel{\beta}{\rightarrow} R^{a}+R^{a}, \\
& S^{a}+R^{d} \stackrel{\beta}{\rightarrow} R^{a}+R^{d}
\end{aligned}
$$

(iii) Inactive spreading

$$
\begin{aligned}
& I^{d}+S^{a} \stackrel{\lambda}{\rightarrow} I^{d}+S^{a} \\
& S^{d}+S^{a} \stackrel{\beta}{\rightarrow} R^{d}+S^{a} \\
& S^{d}+R^{a} \stackrel{\beta}{\rightarrow} R^{d}+R^{a}
\end{aligned}
$$

(iv) Stop spreading a rumor spontaneously

$$
\begin{aligned}
& S^{a} \stackrel{\delta}{\rightarrow} R^{a} \\
& S^{d} \stackrel{\delta}{\rightarrow} R^{d}
\end{aligned}
$$

The rumor spreading process can be shown in Figure 1.

Figure 1 has shown the spreading process which describes the state changings of ignorants and spreaders in complex networks. The rumor spreading process will begin with the 


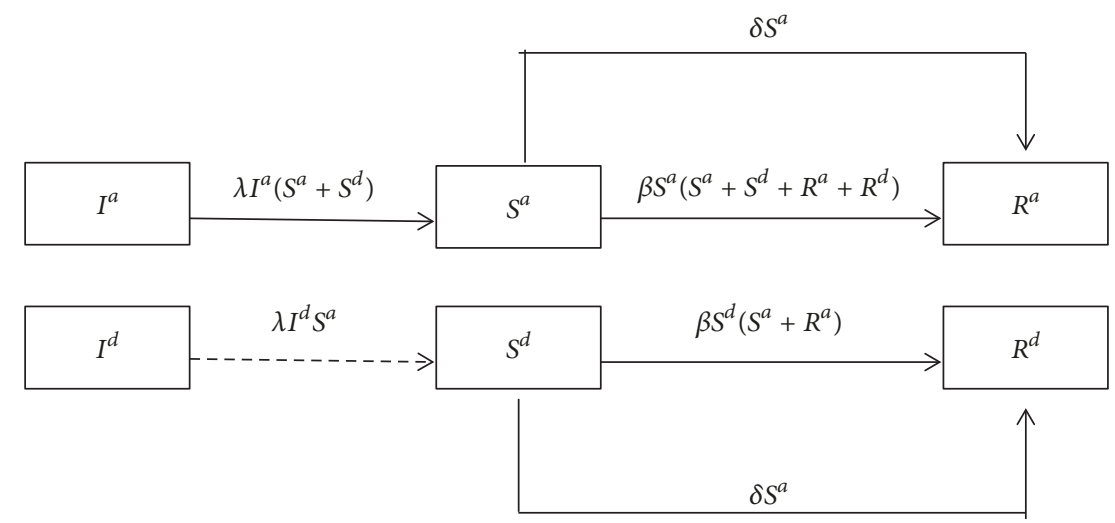

FIGURE 1: Rumor spreading process.

following either situation: (1) an active ignorant node will contact with all spreaders it links to directly and it will become an active spreader with probability $\lambda$. Then the active spreader will meet with another active spreader node, inactive spreader node, active stifler node, or inactive stifler node; the former one will turn into an active stifler node with probability $\beta$ owing to the loss of interest. (2) An inactive node meets with an active spreader and interacts with it; then the active node will become an inactive spreader one with probability $\lambda$. Then the inactive spreader only meets with another active spreader or active stifler node; it will become an inactive stifler node with the same probability $\beta$ due to the forgetting mechanism. Furthermore, considering forgetting mechanism, active spreaders and inactive spreaders can switch their states into active stiflers and inactive stiflers, respectively, at rate $\delta$; it is the rate to stop spreading of a rumor spontaneously

By introducing activeness to ignorant, spreader, and stifler nodes of networks, traditional ISR rumor spreading model has been extended owing to such spreading dynamics.

\section{Mean-Field Approach Analysis}

In this part, we will give the mean field equations of the rumor spreading model on networks. To give mean-field analysis, all nodes are divided into different classes according to their degree following the conventional approach of spreading dynamic study [32]. $N_{k}$ represent the number of nodes in class $k$. Then $I_{k}^{a}(t), I_{k}^{d}(t), S_{k}^{a}(t), S_{k}^{d}(t), R_{k}^{a}(t), R_{k}^{d}(t)$ represent the densities of nodes with degree $k$ at time $t$ in active ignorant, inactive ignorant, active spreader, inactive spreader, active stifler, and inactive stifler state. As we previously assumed that an ignorant node in any degree class will be in active state with rate $\alpha$ and in inactive state with rate $1-$ $\alpha$. Ignoring the interaction between the neighbors of nodes during $[t, t+\Delta t]$, the state transformation can be in the following two situations.

In active ignorant state, node $i$ can interact with any adjacent spreader node with probability $\lambda$. Given that node $i$ has $g(g \leq k)$ adjacent spreaders at time $t$, then the probability of node $i$ keeping in ignorant state wil be $(1-\lambda \Delta t)^{g}$. The probabilities that node $i$ has $g(g \leq k)$ adjacent spreaders at time $t$ will be

$$
N_{i}^{S}(g, t)=C_{k}^{g} \theta(k, t)^{g}[1-\theta(k, t)]^{k-g}
$$

where $\theta(k, t)=\sum_{k^{\prime}} P\left(k^{\prime} \mid k\right) S\left(k^{\prime}, t\right)$ represents the probability of an edge of node $i$ with degree $k$ linking nodes representing spreaders at time $t, P\left(k^{\prime} \mid k\right)$ is the probability of a node with degree $k$ linking to a node with degree $k^{\prime}$, and then the transition probabilities of an active ignorant node at time $t$ for all possible values of $g$ are

$$
\begin{aligned}
& t_{I I}^{a}(k, t)=\sum_{g=0}^{k} C_{k}^{g}(1-\lambda \Delta t)^{g} \theta(k, t)^{g}[1-\theta(k, t)]^{k-g} \\
& t_{I S}^{a}(k, t)=1-t_{I I}^{a}(k, t)
\end{aligned}
$$

In inactive ignorant state, node $i$ can only interacts with any adjacent spreader node. We denote $g(g \leq k)$ as the number of adjacent spreaders of node $i$ at time $t$. Then the probabilities that node $i$ has $g(g \leq k)$ active adjacent spreaders at time $t$ will be

$$
N_{i}^{S}(g, t)=C_{k}^{g}(\alpha \theta(k, t))^{g}[1-\alpha \theta(k, t)]^{k-g}
$$

Similarly, the transition probabilities of an inactive ignorant node at time $t$ for arbitrary $g$ will be

$$
\begin{aligned}
& t_{I I}^{d}(k, t) \\
& \quad=\sum_{g=0}^{k} C_{k}^{g}(1-\lambda \Delta t)^{g}(\alpha \theta(k, t))^{g}[1-\alpha \theta(k, t)]^{k-g} \\
& t_{I S}^{d}(k, t)=1-t_{I I}^{a}(k, t)
\end{aligned}
$$

Based on the above analysis, the probability of an ignorant node $i$ stay in its ignorant state at time $t$ is

$$
\begin{aligned}
t_{I I} & =\alpha t_{I I}^{a}+(1-\alpha) t_{I I}^{d} \\
& =\alpha[1-\lambda \Delta t \theta(k, t)]^{k}+(1-\alpha)[1-\alpha \lambda \Delta t \theta(k, t)]^{k}
\end{aligned}
$$




$$
\begin{aligned}
= & \alpha\left[1-\lambda \Delta t \sum_{k^{\prime}} P\left(k^{\prime} \mid k\right) S\left(k^{\prime}, t\right)\right]^{k} \\
& +(1-\alpha)\left[1-\alpha \lambda \Delta t \sum_{k^{\prime}} P\left(k^{\prime} \mid k\right) S\left(k^{\prime}, t\right)\right]^{k}
\end{aligned}
$$

Similarly, we can deduce the expression for the probability $t_{s s}$ that spreader keep its state unchanged during $[t, t+\Delta t]$. However in this case, we also need to compute the probability of spreader $t$ with degree $k$ linking nodes representing stiflers at time $t$. Following the steps above, we can get

$$
\begin{aligned}
t_{S S} & =\alpha t_{S S}^{a}+(1-\alpha) t_{S S}^{d} \\
& =\alpha\left[1-\beta \Delta t \sum_{k^{\prime}} P\left(k^{\prime} \mid k\right)\left(S\left(k^{\prime}, t\right)+R\left(k^{\prime}, t\right)\right)\right]^{k} \\
& +(1-\alpha) \\
& \cdot\left[1-\alpha \beta \Delta t \sum_{k^{\prime}} P\left(k^{\prime} \mid k\right)\left(S\left(k^{\prime}, t\right)+R\left(k^{\prime}, t\right)\right)\right]^{k}
\end{aligned}
$$

By using the transition probability of (12), the transition rate of ignorant nodes of k-degree class during $[t, t+\Delta t]$ can be expressed as

$$
\begin{aligned}
& I(k, t+\Delta t)=I(k, t)-I(k, t)\left(1-t_{I I}\right)=I(k, t) \\
& -\alpha I(k, t) \\
& \quad \cdot\left[1-\left(1-\lambda \Delta t \sum_{k^{\prime}} P\left(k^{\prime} \mid k\right) S\left(k^{\prime}, t\right)\right)^{k}\right] \\
& -(1-\alpha) I(k, t) \\
& .\left[1-\left(1-\alpha \lambda \Delta t \sum_{k^{\prime}} P\left(k^{\prime} \mid k\right) S\left(k^{\prime}, t\right)\right)^{k}\right]
\end{aligned}
$$

Similarly, we can get the transition rate of spreader nodes and stifler nodes during $[t, t+\Delta t]$ as well:

$$
\begin{aligned}
& S(k, t+\Delta t)=S(k, t)+\alpha I(k, t)[1 \\
& \left.-\left(1-\lambda \Delta t \sum_{k^{\prime}} P\left(k^{\prime} \mid k\right) S\left(k^{\prime}, t\right)\right)^{k}\right]+(1-\alpha) \\
& \cdot I(k, t)\left[1-\left(1-\alpha \lambda \Delta t \sum_{k^{\prime}} P\left(k^{\prime} \mid k\right) S\left(k^{\prime}, t\right)\right)^{k}\right] \\
& -\alpha S(k, t)[1
\end{aligned}
$$

$$
\begin{aligned}
& -\left(1-\beta \Delta t \sum_{k^{\prime}} P\left(k^{\prime} \mid k\right)\left(S\left(k^{\prime}, t\right)+R\left(k^{\prime}, t\right)\right)^{k}\right. \\
& \cdot(1-\delta \Delta t)]-(1-\alpha) S(k, t)[1
\end{aligned}
$$$$
\begin{aligned}
& -\left(1-\alpha \beta \Delta t \sum_{k^{\prime}} P\left(k^{\prime} \mid k\right)\left(S\left(k^{\prime}, t\right)+R\left(k^{\prime}, t\right)\right)^{k}\right. \\
& \cdot(1-\delta \Delta t)]
\end{aligned}
$$

$$
\begin{aligned}
& R(k, t+\Delta t)=\alpha S(k, t)[1 \\
& -\left(1-\beta \Delta t \sum_{k^{\prime}} P\left(k^{\prime} \mid k\right)\left(S\left(k^{\prime}, t\right)+R\left(k^{\prime}, t\right)\right)^{k}\right. \\
& \cdot(1-\delta \Delta t)]+(1-\alpha) S(k, t)[1 \\
& -\left(1-\alpha \beta \Delta t \sum_{k^{\prime}} P\left(k^{\prime} \mid k\right)\left(S\left(k^{\prime}, t\right)+R\left(k^{\prime}, t\right)\right)^{k}\right. \\
& \cdot(1-\delta \Delta t)]
\end{aligned}
$$

Equations (13)-(15) consist of a nonlinear dynamical system in which the stable state implies that $S(k, t)=1$ Therefore, we can linearize (12) and (13) around $S(k, t)=0$ by removing all higher-order terms. Then, in the limit $\Delta t \longrightarrow 0$, we can obtain

$$
\begin{aligned}
& \frac{d I_{k}(t)}{d t}=-\left(2 \alpha-\alpha^{2}\right) \lambda k I_{k}(t) \sum_{k^{\prime}} S_{k^{\prime}}(t) p\left(k^{\prime} \mid k\right) \\
& \frac{d S_{k}(t)}{d t}=\left(2 \alpha-\alpha^{2}\right) \lambda k I_{k}(t) \sum_{k^{\prime}} S_{k^{\prime}}(t) p\left(k^{\prime} \mid k\right) \\
& -\left(2 \alpha-\alpha^{2}\right) \beta k S_{k}(t) \sum_{k^{\prime}}\left(S_{k^{\prime}}(t)+R_{k^{\prime}}(t)\right) p\left(k^{\prime} \mid k\right) \\
& -\delta S_{k}(t) \\
& \frac{d R_{k}(t)}{d t}=\left(2 \alpha-\alpha^{2}\right) \beta k S_{k}(t) \sum_{k^{\prime}}\left(S_{k^{\prime}}(t)+R_{k^{\prime}}(t)\right) \\
& \cdot p\left(k^{\prime} \mid k\right)+\delta S_{k}(t)
\end{aligned}
$$

In real spreading dynamics, heterogeneous distributions always exist in the contact frequency of individuals. In order to reveal this effect, we consider aforementioned model on 
a network with power-law connectivity distribution $P(k) \sim$ $k^{-\gamma}$, whose average degree is denoted as $\langle k\rangle=\sum_{k} k P(k)$. For uncorrelated heterogeneous networks, the degree correlation can be written as [32]

$$
P\left(k^{\prime} \mid k\right)=\frac{k^{\prime} P\left(k^{\prime}\right)}{<k>}
$$

where $P(k)$ is degree distribution function of the network.

\section{Steady-State Analysis}

At the beginning of rumor transmission, nearly no people hear about the rumor, provided that, at first, one active spreader exists in the network and the left are people who are active ignorant individuals and inactive individuals. So the initial condition for rumor transmission is $I(0) \approx 1, S(0) \approx 0$ and $R(0)=0$. Later the quantity of the spreaders firstly increases to the top; then it decreases until it goes down to zero at which point the rumor removes and the system achieves stability. In the final equilibrium state, only ignorants and stiflers are remaining. The final size of rumor will be calculated to weigh the level of rumor influence. Here $R=$ $\lim _{\mathrm{t} \rightarrow \infty} R(t)=R(\infty)$ and it will be deduced below in this section.

4.1. Homogeneous Networks. In order to understand the effect of topological structure on our model, we first consider the homogeneous networks where degree fluctuations are very small and no degree correlation exists [32]. All nodes have the same degree: $I_{k}(t), S_{k}(t)$ and $R_{k}(t)$ become $I(t), S(t)$ and $R(t)$, $\sum_{k^{\prime}} P\left(k^{\prime} \mid k\right)=1$. Therefore, the rumor equations become

$$
\begin{aligned}
\frac{d I(t)}{d t}= & -\left(2 \alpha-\alpha^{2}\right) \lambda\langle k\rangle I(t) S(t) \\
\frac{d S(t)}{d t}= & \left(2 \alpha-\alpha^{2}\right) \lambda\langle k\rangle I(t) S(t) \\
& -\left(2 \alpha-\alpha^{2}\right) \beta\langle k\rangle S(t)[S(t)+R(t)] \\
& -\delta S(t) \\
\frac{d R(t)}{d t}= & \left(2 \alpha-\alpha^{2}\right) \beta\langle k\rangle S(t)[S(t)+R(t)]+\delta S(t)
\end{aligned}
$$

where $<k>$ denotes the average degree of the network.

The final size $R$, investigating the spreading threshold in homogeneous networks, can be obtained by analyzing the mean-field equations (20)-(22). Divide (20) by (22), i.e.,

$$
\begin{aligned}
\frac{d R(t)}{d I(t)} & =\frac{\left(2 \alpha-\alpha^{2}\right) \beta\langle k\rangle S(t)[S(t)+R(t)]+\delta S(t)}{-\left(2 \alpha-\alpha^{2}\right) \lambda\langle k\rangle I(t) S(t)} \\
& =-\frac{\beta}{\lambda I(t)}+\frac{\beta}{\lambda}+\frac{\delta}{-\left(2 \alpha-\alpha^{2}\right) \lambda\langle k\rangle I(t)},
\end{aligned}
$$

that is,

$$
\begin{aligned}
d R(t)= & \frac{\beta}{\lambda} d I(t) \\
& +\left[\frac{\beta}{-\lambda I(t)}+\frac{\delta}{-\left(2 \alpha-\alpha^{2}\right) \lambda\langle k\rangle I(t)}\right] d I(t)
\end{aligned}
$$

Both sides of (20) are integrated for $R(t)$ and $I(t)$ from instability to equilibrium. Noticing when $t=0, I(0) \approx$ $1, S(0) \approx 0$ and $t \longrightarrow \infty, I(\infty)=1-R(\infty)=1-R$, we can get

$$
R=1-e^{-\varepsilon R}
$$

where

$$
\varepsilon=\frac{\left(2 \alpha-\alpha^{2}\right)\langle k\rangle(\lambda+\beta)}{\left(2 \alpha-\alpha^{2}\right) \beta\langle k\rangle+\delta}
$$

Only when $\varepsilon>1$, will (25) get a nonzero solution. For $\delta \neq 0$, the following condition will be satisfied:

$$
\frac{\left(2 \alpha-\alpha^{2}\right)\langle k\rangle \lambda}{\delta}>1
$$

which can be deduced to get the constraint $\lambda>\lambda_{c}$, in that the threshold $\lambda_{c}=\delta /\left(2 \alpha-\alpha^{2}\right)\langle k\rangle$ is associated with homogeneous networks and activity rate.

On the other hand, when the forgetting mechanism is absent, namely $\delta=0$, we can get the equation $\varepsilon=1+\lambda / \beta$; therefore, it can be concluded that (22) always exists as a nonzero solution.

Meanwhile, noting that $\lambda_{c}$ does not always make sense. If $\alpha<1-\sqrt{1-\delta /\langle k\rangle}, \lambda_{c}$ will be greater than 1 . Namely, when the activity rate is under a certain threshold, final transmission scale will be relatively narrow whatever the spreading rate is.

Similarly, threshold of activity rate can be also got from

$$
\alpha_{c}=1-\sqrt{1-\frac{\delta}{\lambda\langle k\rangle}}
$$

which makes sense only when $\lambda>\delta /\langle k\rangle$ for the same reason.

4.2. Heterogeneous Networks. Here, we could express degreedegree correlations as $P\left(k^{\prime} \mid k\right)=k^{\prime} P\left(k^{\prime}\right) /\langle k\rangle=q\left(k^{\prime}\right)$, where $q\left(k^{\prime}\right)$ is the degree distribution function. We assume that initially, the number of all the ignorant nodes could be expressed as $I_{k}(0)=I(0)$. Generally, the density of ignorant nodes can be estimated as $I_{k}(0) \approx 1$. In this case, we can integrate (20) directly and get

$$
I_{k}(t)=e^{-\left(2 \alpha-\alpha^{2}\right) \lambda k \phi(t)}
$$

Then we can introduce such auxiliary function as

$$
\phi(t)=\int_{0}^{t} \sum_{k} S_{k}\left(t^{\prime}\right) q(k) d t^{\prime}=\int_{0}^{t}\left\langle\left\langle S_{k}\left(t^{\prime}\right)\right\rangle\right\rangle d t^{\prime}
$$


And here $\langle\langle O(k)\rangle\rangle=\sum_{k} O(k) q(k)$ has been used. It is necessary to figure out $\phi(\infty)$ to work out the expression of final rumor spreading scale $R$ and here $\phi_{\infty}=\lim _{t \rightarrow \infty} \phi(t)=$ $\phi(\infty)$. Since the initial distribution of ignorants is $I_{k}(0)=$ $I(0)$, when we multiply (17) with $q(k)$ and sum them over $\mathrm{k}$ and then integrate the equation, we can get a differential equation of the density of spreader nodes. After some elementary operations, the following can be obtained:

$$
\begin{aligned}
& \frac{d \phi}{d t} \\
& \quad=1-\left\langle\left\langle e^{-\left(2 \alpha-\alpha^{2}\right) \lambda k \phi}\right\rangle\right\rangle-\left(2 \alpha-\alpha^{2}\right) \beta \\
& \quad * \int_{0}^{t}\left\langle\left\langle k S_{k}\left(t^{\prime}\right)\right\rangle\right\rangle\left(1-\left\langle\left\langle e^{-\left(2 \alpha-\alpha^{2}\right) \lambda k \phi\left(t^{\prime}\right)}\right\rangle\right\rangle\right) d t^{\prime} \\
& \quad-\delta \phi(t)
\end{aligned}
$$

In general, initially, the quantity of ignorant nodes can be expressed as $I(0) \approx 1$.

In the limit $t \longrightarrow \infty, d \phi / d t=0$, then we can get

0

$$
\begin{aligned}
= & 1-\left\langle\left\langle e^{-\left(2 \alpha-\alpha^{2}\right) \lambda k \phi}\right\rangle\right\rangle-\left(2 \alpha-\alpha^{2}\right) \beta \\
& * \int_{0}^{t}\left\langle\left\langle k S_{k}\left(t^{\prime}\right)\right\rangle\right\rangle\left(1-\left\langle\left\langle e^{-\left(2 \alpha-\alpha^{2}\right) \lambda k \phi\left(t^{\prime}\right)}\right\rangle\right\rangle\right) d t^{\prime} \\
& -\delta \phi(t)
\end{aligned}
$$

where $\phi_{\infty}=\lim _{t \rightarrow \infty} \phi(t)$.

For $\beta=0$, it is very easy to solve (32) explicitly to work out $\phi_{\infty}$. For $\beta \neq 0$, we can expand $\beta$ to leading order and integrate (17) to zero order in $\beta$ to solve (32); the following can be reached:

$$
\begin{aligned}
S_{k}(t)= & 1-e^{-\left(2 \alpha-\alpha^{2}\right) \lambda k \phi(t)} \\
& -\delta \int_{0}^{t}\left[1-e^{-\left(2 \alpha-\alpha^{2}\right) \lambda k \phi\left(t^{\prime}\right)}\right] e^{\delta\left(t^{\prime}-t\right)} d t^{\prime} \\
& +O(\beta)
\end{aligned}
$$

Both $\phi(t)$ and $\phi_{\infty}$ are small when the rumor size tends to be in stability state; then we can write $\phi(t)=\phi_{\infty} f(t)$, where the value of $f(t)$ is very small; then expanding $\phi_{\infty}$ to leading order, the following can be reached:

$$
\begin{aligned}
& S_{k}(t) \\
& \quad \simeq\left(2 \alpha-\alpha^{2}\right) \lambda k \phi_{\infty}\left[f(t)-\delta \int_{0}^{t} f\left(t^{\prime}\right) e^{\delta\left(t^{\prime}-t\right)} d t^{\prime}\right] \\
& \quad+O\left(\phi_{\infty}^{2}\right)+O(\beta)
\end{aligned}
$$

Importing (34) into (32) and expanding $\phi_{\infty}$ to the relevant order of the exponential following the conventional approach of spreading dynamic studies [32], the following can be obtained:

$$
\begin{aligned}
0= & \phi_{\infty}\left\{2 \alpha-\alpha^{2}\right) \lambda\langle\langle k\rangle\rangle-\delta \\
& -\left[\left(2 \alpha-\alpha^{2}\right) \lambda\right]^{2}\left\langle\left\langle k^{2}\right\rangle\right\rangle \phi_{\infty} *\left[\frac{1}{2}\right. \\
& \left.+\left(2 \alpha-\alpha^{2}\right) \beta\langle\langle k\rangle\rangle I\right\}+O\left(\beta^{2}\right)+O\left(\phi_{\infty}{ }^{3}\right)
\end{aligned}
$$

where $I=\int_{0}^{\infty}\left[\int_{0}^{t} f\left(t^{\prime}\right) e^{\delta\left(t^{\prime}-t\right)} d t^{\prime}\right] f(t) d t$, an integral, whose value is finite and positive. Obviously, $\phi_{\infty}=0$ works as one solution of the above equation and another nontrivial solution is obtained as

$$
\begin{aligned}
& \phi_{\infty} \\
& =\frac{\left(2 \alpha-\alpha^{2}\right) \lambda\langle\langle k\rangle\rangle-\delta}{\left[\left(2 \alpha-\alpha^{2}\right) \lambda\right]^{2}\left\langle\left\langle k^{2}\right\rangle\right\rangle\left[\frac{1}{2}+\left(2 \alpha-\alpha^{2}\right) \beta\langle\langle k\rangle\rangle I\right]}
\end{aligned}
$$

Note that $\langle\langle k\rangle\rangle=\left\langle k^{2}\right\rangle /\langle k\rangle$, so $\left(2 \alpha-\alpha^{2}\right) \lambda\langle\langle k\rangle\rangle-\delta=$ $\left(2 \alpha-\alpha^{2}\right) \lambda\left(\left\langle k^{2}\right\rangle /\langle k\rangle\right)-\delta>0$, that is, $\lambda>\langle k\rangle \delta /\left(2 \alpha-\alpha^{2}\right)\left\langle k^{2}\right\rangle$, which yields a positive value for $\phi_{\infty}$. That means the threshold of the spreading rumor model in heterogeneous networks is

$$
\lambda_{c}=\frac{\langle k\rangle \delta}{\left(2 \alpha-\alpha^{2}\right)\left\langle k^{2}\right\rangle}
$$

Note that $\lambda_{c}$ does not always make sense. When $\alpha<$ $1-\sqrt{1-\delta\langle k\rangle /\left\langle k^{2}\right\rangle}, \lambda_{c}$ will be greater than 1 . That is to say, when the node activity is under a certain threshold, the transmission scale will be relatively small whatever the spreading rate is. And from this, the threshold of activity rate can be obtained

$$
\alpha_{c}=1-\sqrt{1-\frac{\delta\langle k\rangle}{\lambda\left\langle k^{2}\right\rangle}}
$$

which validates only when $\lambda>\delta\langle k\rangle /\left\langle k^{2}\right\rangle$ for the same reason. Now, from (37) and (38), one can find that the thresholds of transmission rate and activity rate are interdependent. Actually, these two equations represent the same curve. When the propagation dynamics is below the curve, the final transmission scale will be small. From (38), we can find that taking some measures to affect individual activity can also help us to control spreading dynamics.

As $R_{k}(\infty)=1-I_{k}(\infty)$, the final size of the rumor $R$ can be obtained by

$$
R=\sum_{k} P(k)\left(1-e^{-\left(2 \alpha-\alpha^{2}\right) \lambda k \phi(t)}\right)
$$

After importing (36) and developing to the relevant part of the exponential according to [32] the following will be obtained: 


$$
\begin{aligned}
R & \approx \frac{2\left\langle k^{2}\right\rangle^{2}\left(2 \alpha-\alpha^{2}\right) \lambda_{c}}{\left\langle\left\langle k^{2}\right\rangle\right\rangle\left(\langle k\rangle+2\left(2 \alpha-\alpha^{2}\right)\left\langle k^{2}\right\rangle \beta I\right) \delta}\left(1-\frac{\lambda_{c}}{\lambda}\right) \\
& =\frac{2\left\langle k^{2}\right\rangle\langle k\rangle}{\left\langle\left\langle k^{2}\right\rangle\right\rangle\left(\langle k\rangle+2\left(2 \alpha-\alpha^{2}\right)\left\langle k^{2}\right\rangle \beta I\right)}(1 \\
& \left.-\frac{1-\left(1-\alpha_{c}\right)^{2}}{2 \alpha-\alpha^{2}}\right)
\end{aligned}
$$

where $\lambda \geq \lambda_{c}$ and $\alpha \geq \alpha_{c}$.

The former value of $R$ in (40) shows the relation between final transmission scale and the threshold of spreading rate when the node activity is fixed; the latter value of $R$ in (40) shows the relation of transmission scale and the threshold of node activity rate when the spreading rate is fixed. Since (29) has the exponential form, the exponential relations of $R$ and $1 / \lambda$ as well as $R$ and $1 /\left(2 \alpha-\alpha^{2}\right)$ can be speculated. Note that the above relations will not validate until both $\lambda$ and $\alpha$ exist above the curve.

\section{Numerical Simulation}

In this section, theoretical prediction is validated by performing the numerical simulations. As described in Sections 2 and 3 , our studies are mainly focused on homogeneous networks whose average degree $\langle k\rangle=10$ and network size $N=10^{6}$. As far as heterogeneous network is concerned, the power law degree of this network is distributed as $P(k)=(1+$ $\gamma) m^{1+\gamma} k^{-2-\gamma}$, where we set $\gamma=1$ and $m=1$, whose network size $N=10^{6}$, average degree $\langle k\rangle=1.62$, and secondary moment is $\left\langle k^{2}\right\rangle=7.93$. Besides, we assume that initially, only one active spreader exists in the network and the left are people who are active ignorant individuals and inactive individuals. So the initial condition for rumor transmission is $I(0) \approx$ $1, S(0) \approx 0$ and $R(0)=0$.

Firstly, the influence the spreading rate and individual activity rate have on final rumor transmission scale can be found from the heat map in the Figure 2. The color shade represents the final rumor spreading scale. There critical exists phenomenon, specifically, the curve consisting of critical spreading rate and critical activity rate can be clearly seen there. The solid white line in Figure 2 accords with $\lambda=0.205 * 0.6 /\left(2 \alpha-\alpha^{2}\right)$, where $\langle k\rangle /\left\langle k^{2}\right\rangle=0.205$ and $\delta=0.6$, which matches the critical curve well. The horizontal dashed line consists with $\lambda=0.123$, below which the scale of rumor propagation will be relatively small in network. The vertical dashed line corresponds to $\alpha=1-\sqrt{1-\delta\langle k\rangle /\left\langle k^{2}\right\rangle}=$ 0.063 which implies that the final transmission scale will be small even the spreading rate $\lambda$ is up to 1 . Fixing the horizontal axis, we can find that spreading rate has a positive effect on final rumor size. Similarly, fixing the vertical axis, it can be found that activity rate is also positively related to final rumor scale.

To display critical phenomenon of $\lambda$ and $\alpha$ clearer, the final rumor spreading scale as the function of spreading rate $\lambda$ and activity rate $\alpha$ are separately performed in Figures 3 and 4. In Figure 3 , we set $\beta=0.1, \delta=0.6$, and $\alpha=0.3,0.5,0.7$, and

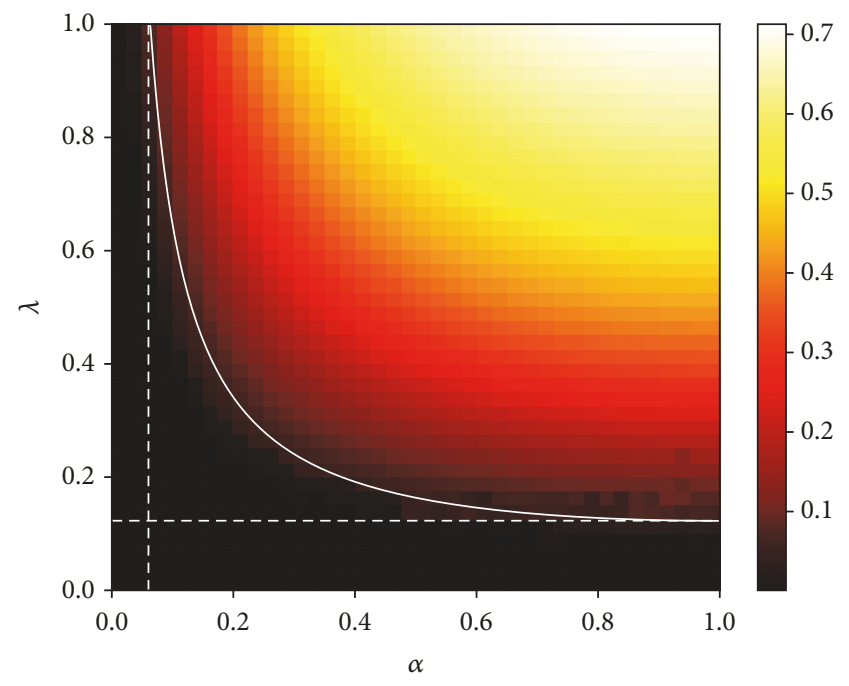

FIGURE 2: Final rumor transmission scale $R$ with different $\alpha$ and $\lambda$. Solid line matches the $\lambda=\langle k\rangle /\left\langle k^{2}\right\rangle * \delta /\left(2 \alpha-\alpha^{2}\right)$. Horizontal dashed line consists with $\lambda=\langle k\rangle /\left\langle k^{2}\right\rangle * \delta$, and vertical dashed line corresponds to $\alpha=1-\sqrt{1-\delta\langle k\rangle /\left\langle k^{2}\right\rangle}$.

0.9. It is found that the threshold of spreading rate (the black arrows denote the critical thresholds of spreading rate under different activity rates) is greatly related to activity rate $\alpha$, which means that the higher the activity rate is, the lower the threshold of spreading rate is in spreading dynamics. Figure 4 displays that the impact of activity rate on critical spreading rate. In the desire to further study spreading process, $R$ is numerically fitted to stretched exponential function of $\lambda$ and $\alpha$ when $\lambda>\lambda_{c}$ and $\alpha>\alpha_{c}$, respectively (see inset of Figures 3 and 4$)$,

$$
\begin{gathered}
R: e^{-C_{1}(\alpha) / \lambda} \\
R: e^{-C_{2}(\lambda) /\left(2 \alpha-\alpha^{2}\right)}
\end{gathered}
$$

where $C_{1}(\alpha)$ are constant values when $\alpha$ is fixed. Similarly, once $\lambda$ is fixed, $C_{2}(\lambda)$ are constant values. The inference of spreading scale of (39) can be validated by these results.

Next, we explore the effect of the network topology on the rumor spreading by fixing $\beta=0.1, \lambda=0.5$, and $\delta=0.6$. Figures 5 and 6 show the evolution of densities of spreaders $S(t)$ and stiflers $R(t)$ under different activity rates. Figure 5 illustrates the densities of spreaders varying under different activity rate in different networks; it can be found that the quantity of spreaders firstly goes up until it reaches peak value, and then it will decrease to zero. On top of that, it is no wonder that when the activity is small, the individuals in the network will not be active to spread rumors; therefore whatever the network is, the increment of spreaders as well as the number of spreaders totally will be very small. What is more, the time the spreading process will last much longer, compared to higher activity rate.

Figure 6 illustrates the densities of stiflers versus time varying different activity rates under both homogeneous and heterogeneous networks. It can be observed that activity rate determines the increment speed as well as final size of 


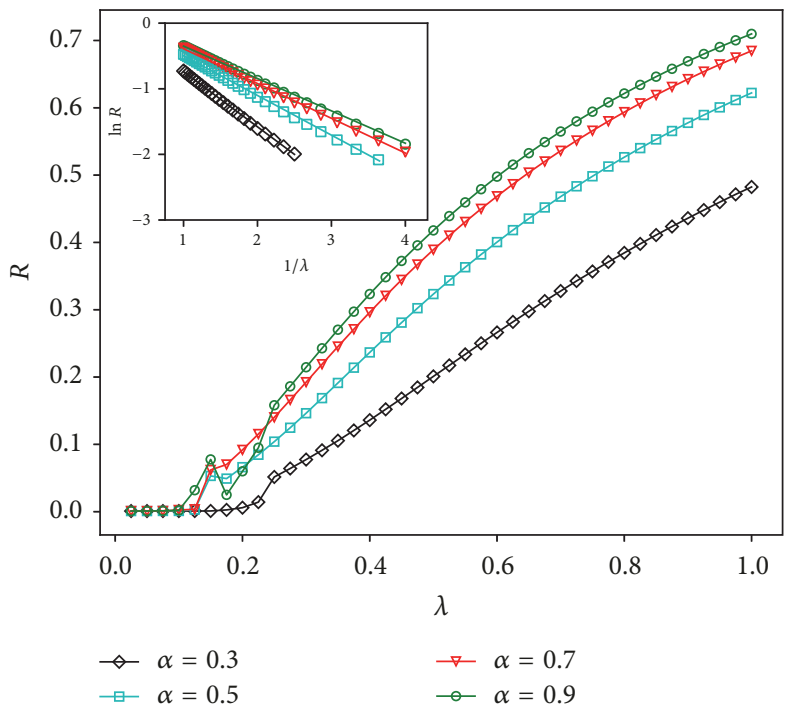

Figure 3: Final rumor size $R$ as a function of spreading rate $\lambda$ varying over different parameter $\alpha$. Insets show the exponential correlation of $R$ and $1 / \lambda$ when $\alpha$ is greater than threshold $\alpha_{c}$.

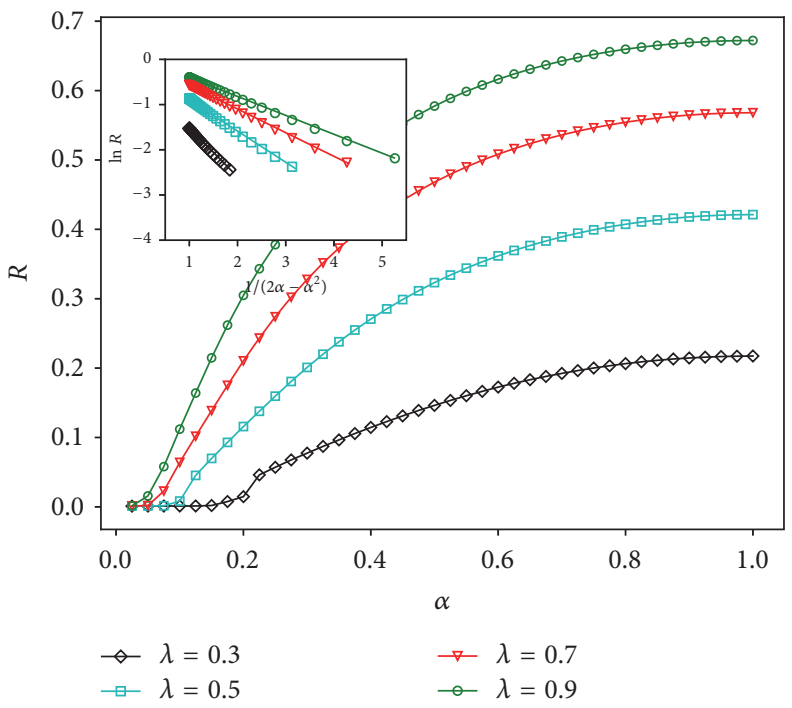

Figure 4: Final rumor size $R$, as a function of activity rate $\alpha$ varying over different $\lambda$. Insets show the exponential correlation of $R$ and $1 /\left(2 \alpha-\alpha^{2}\right)$ when $\lambda$ is greater than critical $\lambda_{c}$.

rumor spreading in large scale, which can be specified as large spreading rate results in large final size of stiflers. Furthermore, it can be observed that topology network and activity rate both exert effect on the time that the spreading process requires to reach steady state. Specifically, the final rumor spreading scale in homogeneous network is much larger, comparing to that in heterogeneous networks, and it needs shorter time to reach steady state in homogeneous networks under the same activity rate.

\section{Conclusion}

This work investigated the rumor spreading process based on ISR with node activity considered both in homogeneous and heterogeneous networks. Different from other scholars, in this model, an activity rate is introduced for each node in the network. An active node can interact with nodes it links to directly, while an inactive node could only interact with the active nodes it links directly to. The behavior of the model is studied and analyzed on both homogeneous and heterogeneous networks by conducting mean-field method. It reveals that taking the node activity and network topology into account, the threshold of rumor spreading size is relevant to both activity rate and spreading rate; besides the threshold rate is positively related to node activity. It can be concluded that when the activity rate is higher, and the final size of rumor spreading will be higher. Furthermore, it is found that comparing to homogeneous networks, not only is spreading scale on heterogeneous networks small but also the transmission 


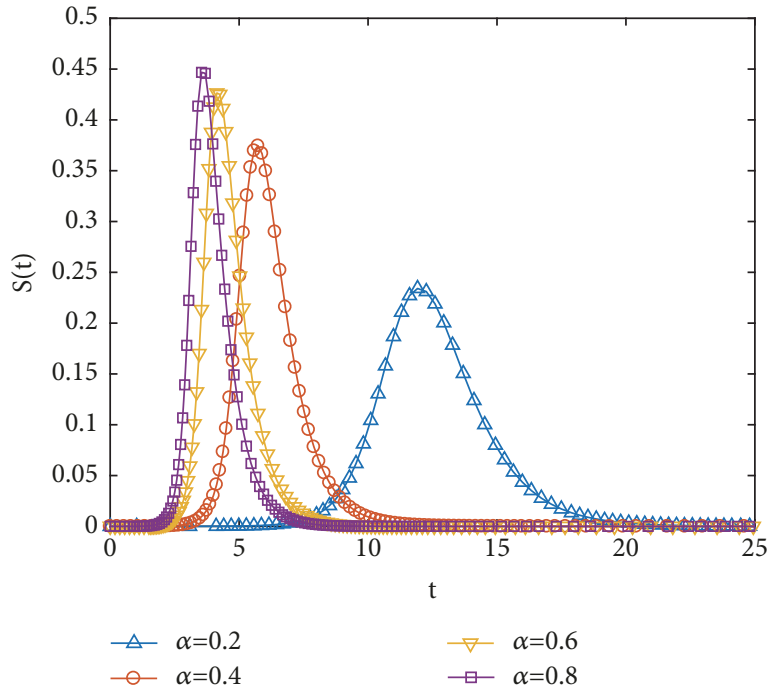

(a) Density of spreaders in homogeneous network

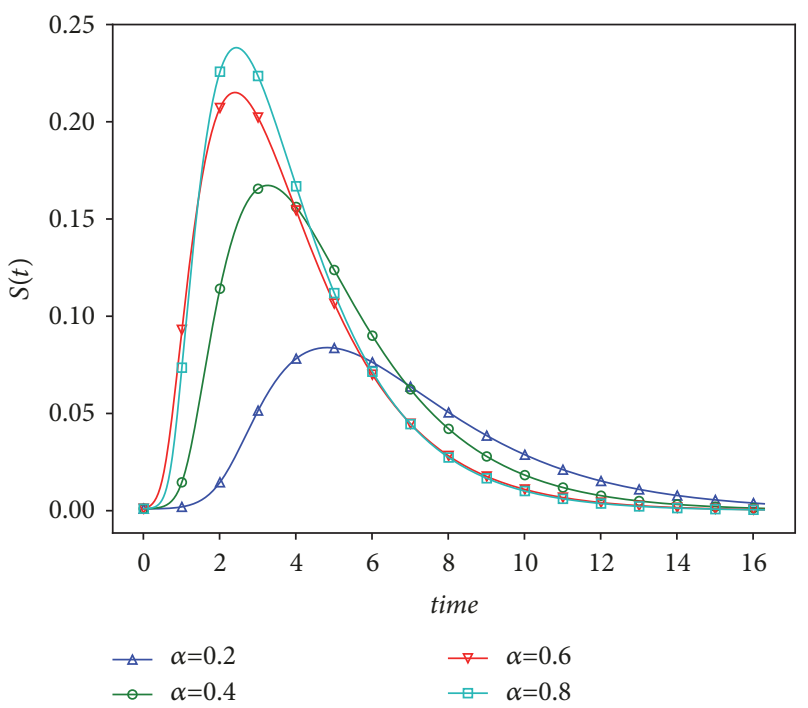

(b) Density of spreaders in heterogeneous network

FIGURE 5: The evolution of spreaders varying over the activity rate $\alpha$ with $\beta=0.1, \lambda=0.5$, and $\delta=0.6$.

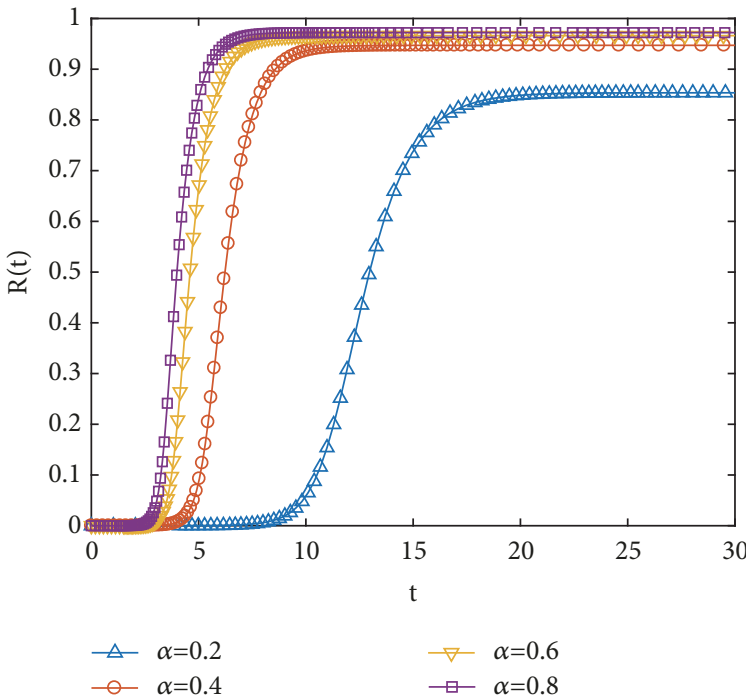

(a) Density of stiflers in homogeneous network

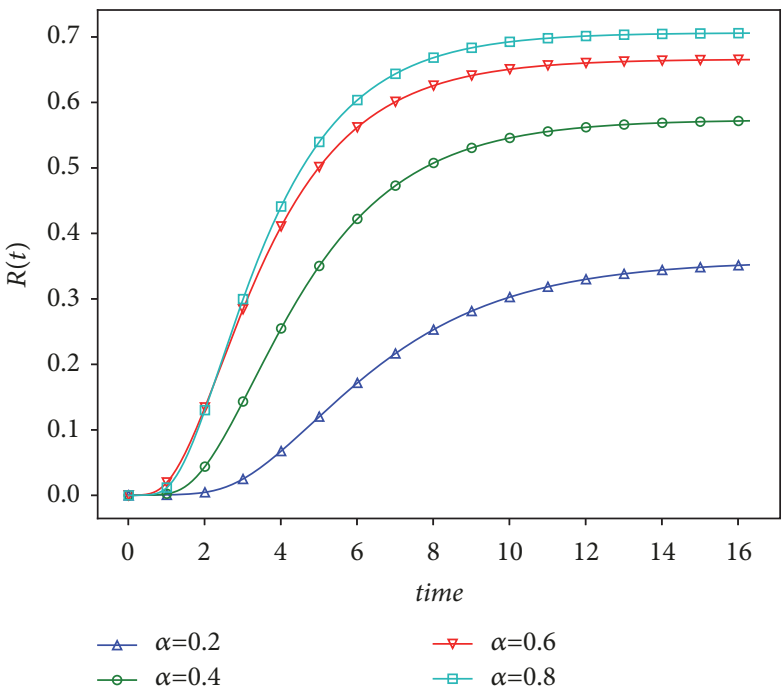

(b) Density of stiflers in heterogeneous network

FIGURE 6: The evolution of stiflers varying over the activity rate $\alpha$ with $\beta=0.1, \lambda=0.5$, and $\delta=0.6$.

speed of rumor on heterogeneous network is much faster. These results have been validated by numerical simulations on both homogeneous and heterogeneous networks. It can be the highlight that our paper established a simple model to analyze the influence of individual activity has on rumor spreading to consider the effect individual behavior have on rumor spreading more directly, comparing to those studies on rumor spreading considering individual behavior pattern. These results can guide us in controlling rumor spreading in real life.

In brief, taking node activity into consideration may help us broaden our vision to figure out more applicable and costefficient methods to stop rumor spreading.

\section{Conflicts of Interest}

The authors declare that there are no conflicts of interest regarding the publication of this article.

\section{Authors' Contributions}

Liang'an Huo conceived of the presented idea. Fan Ding developed the theory and performed the computations. Chen Liu verified the analytical methods. Yingying Cheng analyzed sequencing data and developed analysis tools. All authors discussed the results and contributed to the final manuscript. 


\section{Acknowledgments}

This work partially supported by the Project of Humanity and Social Science foundation of University of Shanghai Science and Technology (SK17ZD02, SK18ZD03), the Project of Fundamental Research Funds for the Central Universities (2017ECNV-KXK016), and the National Natural Science Foundation of China (71774111, 61702331, 71303157). The authors are very grateful to the anonymous referees for their valuable comments and suggestions, helping them to improve the quality of this paper.

\section{References}

[1] S. Galam, "Modelling rumors: the no plane Pentagon French hoax case," Physica A: Statistical Mechanics and its Applications, vol. 320, pp. 571-580, 2003.

[2] Y. Moreno, M. Nekovee, and A. F. Pacheco, "Dynamics of rumor spreading in complex networks," Physical Review E: Statistical, Nonlinear, and Soft Matter Physics, vol. 69, no. 6, Article ID 066130, 2004.

[3] S. Aral and D. Walker, "Creating social contagion through viral product design: A randomized trial of peer influence in networks," Management Science, vol. 57, no. 9, pp. 1623-1639, 2011.

[4] X. Qiu, L. Zhao, J. Wang, X. Wang, and Q. Wang, "Effects of time-dependent diffusion behaviors on the rumor spreading in social networks," Physics Letters A, vol. 380, no. 24, pp. 20542063, 2016.

[5] X. Guardiola, A. Díaz-Guilera, C. J. Pérez, A. Arenas, and M. Llas, "Modeling diffusion of innovations in a social network," Physical Review E: Statistical, Nonlinear, and Soft Matter Physics, vol. 66, no. 2, Article ID 026121, 2002.

[6] R. Pastor-Satorras and A. Vespignani, "Epidemic spreading in scale-free networks," Physical Review Letters, vol. 86, no. 14, pp. 3200-3203, 2001.

[7] Q. Liu, T. Li, and M. Sun, "The analysis of an SEIR rumor propagation model on heterogeneous network," Physica A: Statistical Mechanics and its Applications, vol. 469, pp. 372-380, 2017.

[8] D. J. Daley and D. G. Kendall, "Stochastic rumours," Journal of the Institute of Mathematics and Its Applications, vol. 1, pp. 4255, 1965.

[9] D. J. Daley and J. Gani, Epidemic Modeling: An Introduction, Cambridge University Press, 1999.

[10] D. P. Maki and M. Thompson, Mathematical Models and Applications: With Emphasis on the Social, Life, and Management Sciences, Prentice Hall, Englewood Cliffs, NJ, USA, 1973.

[11] J. D. Murray, Mathematical Modelling in Epidemiology, Springer, Berlin, 1980.

[12] D. S. Callaway, M. E. J. Newman, S. H. Strogatz, and D. J. Watts, "Network robustness and fragility: percolation on random graphs," Physical Review Letters, vol. 85, no. 25, pp. 5468-5471, 2000.

[13] D. J. Watts and S. H. Strogatz, "Collective dynamics of smallworld networks," Nature, vol. 393, pp. 440-442, 1998.

[14] S. H. Strogatz, "Exploring complex networks," Nature, vol. 410, no. 6825 , pp. 268-276, 2001.

[15] D. H. Zanette, "Critical behavior of propagation on small-world networks," Physical Review E: Statistical, Nonlinear, and Soft Matter Physics, vol. 64, no. 5, 2001.
[16] D. H. Zanette, "Dynamics of rumor propagation on small-world networks," Physical Review E: Statistical, Nonlinear, and Soft Matter Physics, vol. 65, no. 4, Article ID 041908, 9 pages, 2002.

[17] Y. Moreno, M. Nekovee, and A. Vespignani, "Efficiency and reliability of epidemic data dissemination in complex networks," Physical Review E: Statistical, Nonlinear, and Soft Matter Physics, vol. 69, Article ID 055101(R), 2004.

[18] Y. Moreno, R. Pastor-Satorras, and A. Vespignani, "Epidemic outbreaks in complex heterogeneous networks," The European Physical Journal B, vol. 26, no. 4, pp. 521-529, 2002.

[19] G. Csányi and B. Szendrői, "Structure of a large social network," Physical Review E: Statistical, Nonlinear, and Soft Matter Physics, vol. 69, no. 3, 2004.

[20] H. Ebel, L.-I. Mielsch, and S. Bornholdt, "Scale-free topology of e-mail networks," Physical Review E: Statistical, Nonlinear, and Soft Matter Physics, vol. 66, no. 3, Article ID 035103, 2002.

[21] S. Boccaletti, V. Latora, Y. Moreno, M. Chavez, and D. W. Hwang, "Complex networks: Structure and dynamics," Physics Reports, vol. 424, no. 4-5, pp. 175-308, 2006.

[22] J. Zhou, Z. Liu, and B. Li, "Influence of network structure on rumor propagation," Physics Letters A, vol. 368, no. 6, pp. 458463, 2007.

[23] S. Deng and W. Li, "Spreading dynamics of forget-remember mechanism," Physical Review E: Statistical, Nonlinear, and Soft Matter Physics, vol. 95, no. 4, 2017.

[24] L. Huo, L. Wang, and G. Song, "Global stability of a twomediums rumor spreading model with media coverage," Physica A: Statistical Mechanics and its Applications, vol. 482, pp. 757-771, 2017.

[25] L.-L. Xia, G.-P. Jiang, B. Song, and Y.-R. Song, "Rumor spreading model considering hesitating mechanism in complex social networks," Physica A: Statistical Mechanics and its Applications, vol. 437, pp. 295-303, 2015.

[26] D. Li and J. Ma, "How the government's punishment and individual's sensitivity affect the rumor spreading in online social networks," Physica A: Statistical Mechanics and its Applications, vol. 469, pp. 284-292, 2017.

[27] L. Huo, L. Wang, N. Song, C. Ma, and B. He, "Rumor spreading model considering the activity of spreaders in the homogeneous network," Physica A: Statistical Mechanics and its Applications, vol. 468, pp. 855-865, 2017.

[28] J. L. Iribarren and E. Moro, "Impact of Human Activity Patterns on the Dynamics of Information Diffusion," Physical Review Letters, vol. 103, no. 3, 2009.

[29] B. Min, K. Goh, and A. Vazquez, "Spreading dynamics following bursty human activity patterns," Physical Review E: Statistical, Nonlinear, and Soft Matter Physics, vol. 83, no. 3, 2011.

[30] S. Liu, N. Perra, M. Karsai, and A. Vespignani, "Controlling Contagion Processes in Activity Driven Networks," Physical Review Letters, vol. 112, no. 11, 2014.

[31] C. Liu, L.-x. Zhou, C.-j. Fan, L.-a. Huo, and Z.-w. Tian, "Activity of nodes reshapes the critical threshold of spreading dynamics in complex networks," Physica A: Statistical Mechanics and its Applications, vol. 432, pp. 269-278, 2015.

[32] M. Nekovee, Y. Moreno, G. Bianconi, and M. Marsili, “Theory of rumour spreading in complex social networks," Physica A: Statistical Mechanics and its Applications, vol. 374, no. 1, pp. 457470, 2007. 


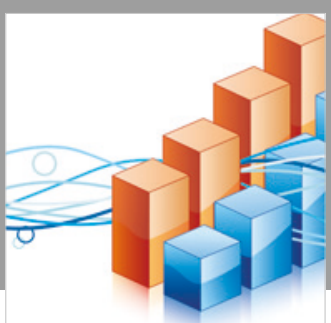

Advances in

Operations Research

\section{-n-m}
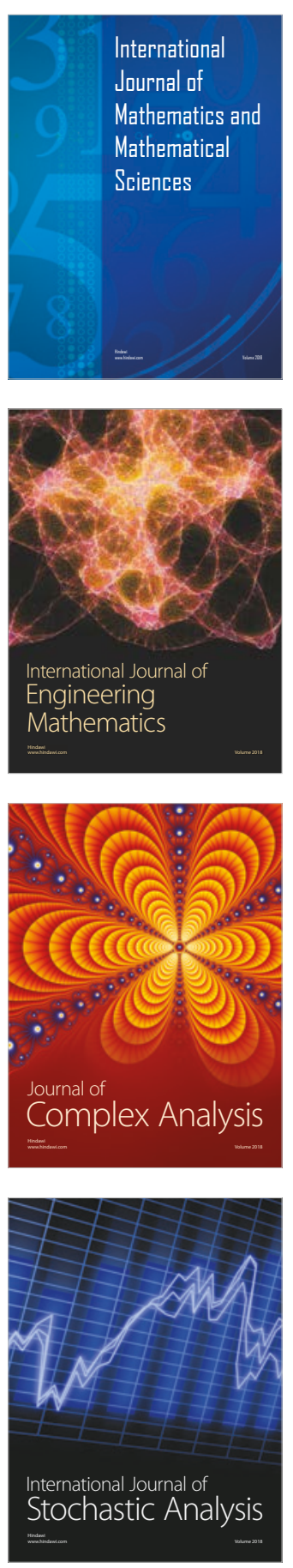
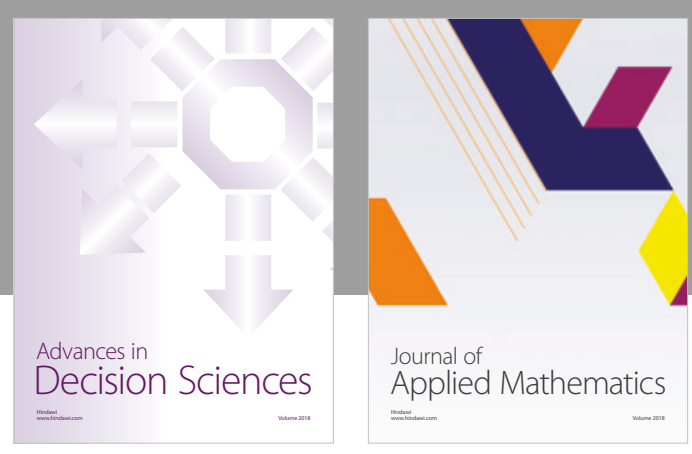

Journal of

Applied Mathematics
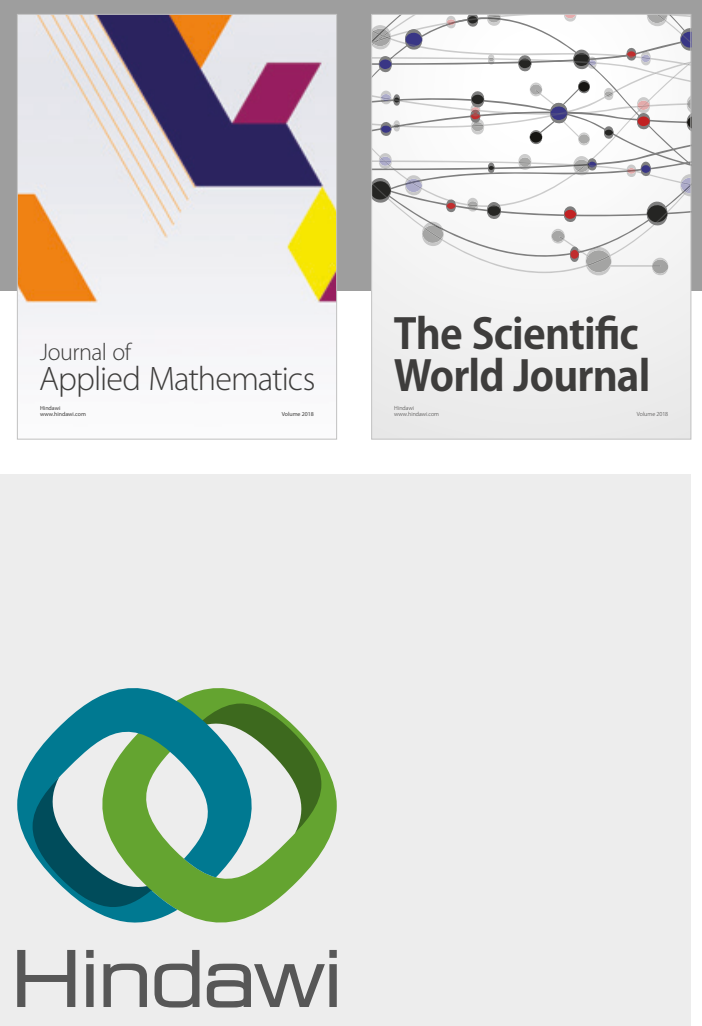

Submit your manuscripts at

www.hindawi.com

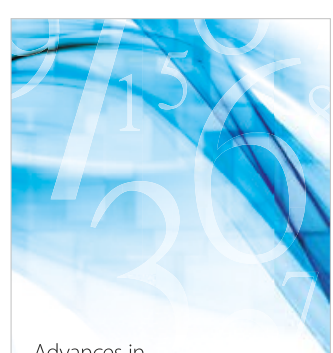

Advances in
Numerical Analysis
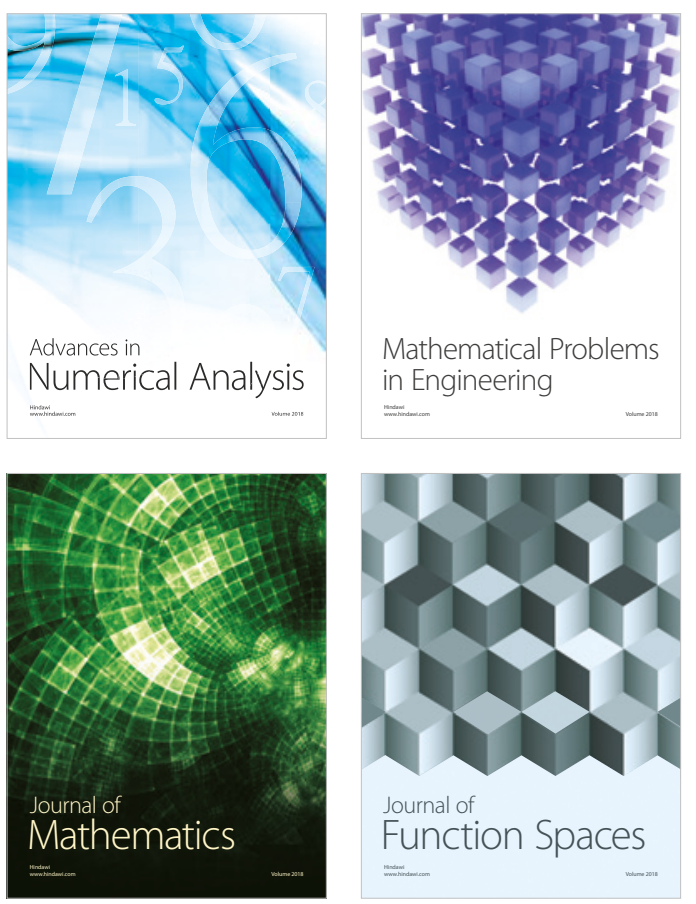

Mathematical Problems in Engineering

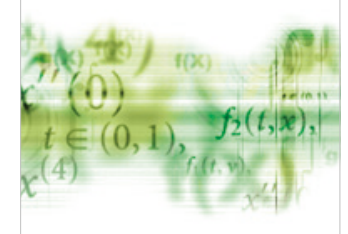

International Journal of

Differential Equations

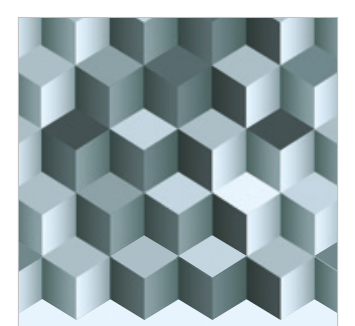

Journal of

Function Spaces
The Scientific

World Journal

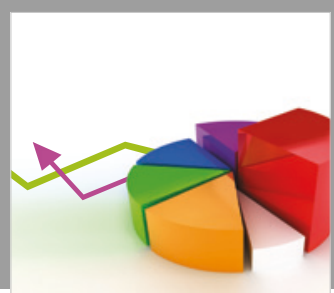

Journal of

Probability and Statistics
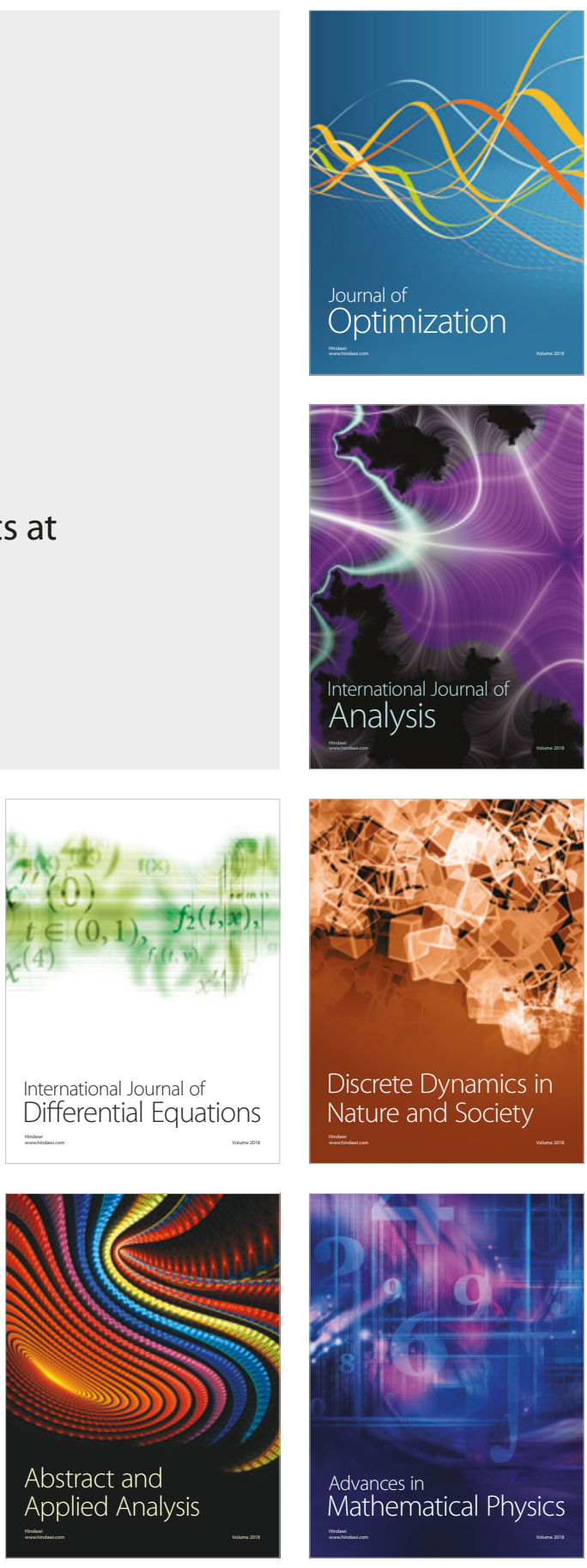